\title{
On a Class of Solvable Difference Equations
}

\author{
Stevo Stević, ${ }^{1,2}$ Mohammed A. Alghamdi, ${ }^{2}$ Naseer Shahzad, ${ }^{2}$ and Dalal A. Maturi ${ }^{3}$ \\ ${ }^{1}$ Mathematical Institute of the Serbian Academy of Sciences, Knez Mihailova 36/III, 11000 Beograd, Serbia \\ ${ }^{2}$ Department of Mathematics, King Abdulaziz University, P.O. Box 80203, Jeddah 21589, Saudi Arabia \\ ${ }^{3}$ Department of Mathematics, Faculty of Science, King Abdulaziz University, P.O. Box 42641, Jeddah 21551, Saudi Arabia
}

Correspondence should be addressed to Stevo Stević; sstevic@ptt.rs

Received 25 September 2013; Accepted 22 October 2013

Academic Editor: Josef Diblík

Copyright (C) 2013 Stevo Stević et al. This is an open access article distributed under the Creative Commons Attribution License, which permits unrestricted use, distribution, and reproduction in any medium, provided the original work is properly cited.

A class of difference equations which can be solved in closed form is presented in this paper. Obtained formulas for the solutions are used in the study of the asymptotic behavior of their solutions in a particular case.

\section{Introduction}

One of the interesting problems in the theory of difference equations is to find the equations which can be solved in closed form. Such kind of equations are among the first ones which attracted attention of scientists. The reason for this is a natural expectation that obtained formulas for solutions can considerably facilitate investigation of their long-term behavior. For some classical results in the area, see, for example, book [1]. In [2], Stević solved, in an elegant way, the difference equation:

$$
x_{n+1}=\frac{b x_{n-1}}{b+c x_{n} x_{n-1}}, \quad n \in \mathbb{N}_{0}
$$

explaining the formula in [3], which was given without any theoretical explanation. Several authors later considered some generalizations of (1) mostly by using Stevićs ideas and methods in [2] (see, e.g., [4-9] and the references therein). The idea for solving (1) was to reduce it to a nonhomogeneous linear first-order difference equation, which is solvable. Many equations and systems can be solved in a similar way (see, e.g., $[4,6-12])$. For some related results and applications of linear first-order difference inequalities, see, for example, [13-15].

An interesting difference equation is the following:

$$
x_{n}=\frac{x_{n-k} x_{n-l}}{a x_{n-m}+b x_{n-s}}, \quad n \in \mathbb{N}_{0},
$$

where $k, l, m$, and $s$ are fixed natural numbers, $a, b \in \mathbb{R} \backslash\{0\}$, and the initial values $x_{-i}, i=\overline{1, \tau}, \tau:=\max \{k, l, m, s\}$ are real numbers.

Special cases of (2) frequently appear in the literature.

Example 1. The next equation

$$
x_{n}=\frac{x_{n-1} x_{n-2}}{2 x_{n-1}+x_{n-2}}, \quad n \in \mathbb{N}_{0}
$$

is one of them.

Equation (3) is a special case of (2) with

$$
k=m, \quad l=s,
$$

or

$$
k=s, \quad l=m,
$$

that is, of the equations

$$
\begin{array}{ll}
x_{n}=\frac{x_{n-k} x_{n-l}}{a x_{n-k}+b x_{n-l}}, & n \in \mathbb{N}_{0}, \\
x_{n}=\frac{x_{n-k} x_{n-l}}{a x_{n-l}+b x_{n-k}}, & n \in \mathbb{N}_{0} .
\end{array}
$$

Now note that (6) and (7) share a common important feature. Namely, assume that $\left(x_{n}\right)_{n \geq-\tau}$ is a well-defined solution of (6) or (7) (i.e., all its terms exist and are finite numbers). 
Then, if $x_{n} \neq 0$, for every $n \geq-\tau$, by using the change of variables

$$
y_{n}=\frac{1}{x_{n}}
$$

(6) and (7) are, respectively, transformed into the following linear difference equations with constant coefficients:

$$
\begin{array}{ll}
y_{n}=a y_{n-l}+b y_{n-k}, & n \in \mathbb{N}_{0}, \\
y_{n}=b y_{n-l}+a y_{n-k}, & n \in \mathbb{N}_{0},
\end{array}
$$

which can be solved in closed form. As a consequence, (6) and (7), can be also solved in closed form. In fact, due to the known fact that polynomials of degree five and more need not be explicitly solved, we know that (9) and (10) can be theoretically solved. Nevertheless, we know some methods of how theoretically solutions of (9) and (10) can be found, as well as their form.

Note that for (3) the corresponding equation (9) is

$$
y_{n}=y_{n-1}+2 y_{n-2}
$$

The general solution of (11) is $y_{n}=c_{1} 2^{n}+c_{2}(-1)^{n}$, so that

$$
x_{n}=\frac{1}{c_{1} 2^{n}+c_{2}(-1)^{n}} .
$$

A natural question and a corresponding problem are as follows.

Question 2. Are there any other values of $k, l, m$, and $s$ except those ones in (4) and (5), such that (2) is solvable in closed form?

Problem 3. In the affirmative case find their solutions in closed form.

The answer to the question is positive, and in recent paper [12], as a byproduct of our results therein, we have essentially shown that, in the case

$$
m=k, \quad s=k+l,
$$

(2) is solvable in closed form, and by using the described method therein, in some subcases of the case in (13) we have presented formulas for their solutions and use them in the study of their long-term behavior.

Here we continue this line of research by showing that there are some cases different from those ones in (4), (5), and (13), for which (2) is solvable in closed form.

\section{Case $l=m=k+s$}

In this section we consider (2) in the case

$$
l=m=k+s .
$$

That is, we consider the next difference equation:

$$
x_{n}=\frac{x_{n-k} x_{n-k-s}}{a x_{n-k-s}+b x_{n-s}}, \quad n \in \mathbb{N}_{0} .
$$

First note that we may assume

$$
\operatorname{gcd}(k, s)=1
$$

(the greatest common divisor of natural numbers $k$ and $s$ ). Otherwise, if $\operatorname{gcd}(k, s)=r>1$, then $k=r k_{1}$ and $s=r s_{1}$ for some $k_{1}, s_{1} \in \mathbb{N}$ such that $\operatorname{gcd}\left(k_{1}, s_{1}\right)=1$. Since every $n \in \mathbb{N}_{0}$ has the form $n=m r+i$, for some $m \in \mathbb{N}_{0}$ and $i=\overline{0, r-1}$, (15) can be written in the form

$$
x_{m r+i}=\frac{x_{r\left(m-k_{1}\right)+i} x_{r\left(m-k_{1}-s_{1}\right)+i}}{a x_{r\left(m-k_{1}-s_{1}\right)+i}+b x_{r\left(m-s_{1}\right)+i}} .
$$

Using the next change of variables

$$
x_{t}^{(i)}=x_{t r+i},
$$

$t \geq-\left(k_{1}+s_{1}\right), i=\overline{0, r-1}$, in (17) we have that $\left(x_{t}^{(i)}\right)_{t \geq-\left(k_{1}+s_{1}\right)}$, $i=\overline{0, r-1}$, are $r$ independent solutions of the system

$$
x_{t}=\frac{x_{t-k_{1}} x_{t-k_{1}-s_{1}}}{a x_{t-k_{1}-s_{1}}+b x_{t-s_{1}}}, \quad t \in \mathbb{N}_{0},
$$

which is (15) with $k_{1}$ and $s_{1}$ instead of $k$ and $s$.

Hence, from now on we will assume that condition (16) holds.

2.1. A Case When Some of Initial Values Are Equal to Zero. Now assume that one of the initial values $x_{-i}, i=\overline{1, k+s}$, is equal to zero. If $x_{-i}=0$ for some $1 \leq i \leq k$, then $x_{k-i}$ is not defined or $x_{k-i}=0$. If the later holds, then $a x_{-i}+b x_{k-i}=$ 0 , which along with (15) implies that $x_{k+s-i}$ is not defined. Further, if $x_{-i}=0$ for some $k+1 \leq i \leq k+s$, then $x_{k+s-i}$ is not defined or $x_{k+s-i}=0$. If the later holds, then $x_{2 k+s-i}$ is not defined or $x_{2 k+s-i}=0$. If $x_{2 k+s-i}=0$, then $a x_{k+s-i}+b x_{2 k+s-i}=$ 0 , which along with (15) implies that $x_{2 k+2 s-i}$ is not defined.

On the other hand, if $x_{n_{0}}=0$ for some $n_{0} \in \mathbb{N}_{0}$, then from (15) we see that $x_{n_{0}-k}=0$ or $x_{n_{0}-k-s}=0$. If $x_{n_{0}-k}=0$, then using again (15) we see that $x_{n_{0}-2 k}=0$ or $x_{n_{0}-2 k-s}=0$, while if $x_{n_{0}-k-s}=0$, then $x_{n_{0}-2 k-s}=0$ or $x_{n_{0}-2 k-2 s}=0$. Repeating the procedure we see that there is an $i_{0} \in\{1, \ldots, k+s\}$ such that $x_{-i_{0}}=0$.

From all these considerations it follows that all solutions of (15) such that $x_{-i}=0$ for some $i \in\{1, \ldots, k+s\}$ are not well defined and that if a solution of (15) has a zero term then it is not defined.

Hence, from now on we will consider only those solutions of (15) such that $x_{n} \neq 0$, for every $n \geq-(k+s)$.

2.2. A Case When None of Initial Values Is Equal to Zero. Assume that $\left(x_{n}\right)_{n \geq-(k+s)}$ is a solution of (15) such that $x_{-i} \neq 0$ for every $i \in\{1, \ldots, k+s\}$. Since in this case $x_{n} \neq 0$ for every $n \geq-(k+s)$, then (15) can be written in the following form:

$$
\frac{x_{n}}{x_{n-k}}=\frac{1}{a+b\left(x_{n-s} / x_{n-k-s}\right)}, \quad n \in \mathbb{N}_{0} .
$$

The form suggests the next, natural, change of variables:

$$
y_{n}=\frac{x_{n}}{x_{n-k}}, \quad n \geq-s,
$$


which transforms (20) into the next difference equation of order $s$ :

$$
y_{n}=\frac{1}{a+b y_{n-s}}, \quad n \in \mathbb{N}_{0}
$$

In fact (22) is essentially an equation of the first order. Indeed, if we use the following decomposition of indices $n \rightarrow$ $n s+j, j=\overline{0, s-1},(22)$ can be written as

$$
y_{n}^{(j)}=\frac{1}{a+b y_{n-1}^{(j)}}, \quad n \in \mathbb{N}_{0},
$$

where

$$
y_{n}^{(j)}=y_{n s+j}, \quad n \geq-1, \quad j=\overline{0, s-1}
$$

This means that the subsequences $\left(y_{n s+j}\right)_{n \geq-1}, j=\overline{0, s-1}$, are $s$ independent solutions of the difference equation

$$
z_{n}=\frac{1}{a+b z_{n-1}}, \quad n \in \mathbb{N}_{0}
$$

of the first order.

Equation (25) is solvable, which along with (21) shows that (15) is solvable, giving another positive answer to Question 2. Indeed, (25) is a special case of the next difference equation:

$$
z_{n}=\frac{\alpha z_{n-1}+\beta}{\gamma z_{n-1}+\delta}, \quad n \in \mathbb{N}_{0} .
$$

Equation (26) is classical and can be solved in several ways. Since in the case $\gamma=0(26)$ is a linear first-order difference equation, which is solvable, from now on we will assume that $\gamma \neq 0$. By using the change of variables

$$
z_{n}=\frac{u_{n+1}}{u_{n}}+c, \quad n \in \mathbb{N}_{0}
$$

into (26), after some calculations, we get

$$
\begin{aligned}
& (\gamma c-\alpha) \frac{u_{n}}{u_{n-1}}+\gamma \frac{u_{n+1}}{u_{n-1}}+(\gamma c+\delta) \frac{u_{n+1}}{u_{n}}+c(\gamma c+\delta)-\alpha c-\beta \\
& =0 .
\end{aligned}
$$

By choosing

$$
c=-\frac{\delta}{\gamma}
$$

(here we use the assumption $\gamma \neq 0$ ) we get

$$
\gamma u_{n+1}-(\alpha+\delta) u_{n}+\frac{\alpha \delta-\beta \gamma}{\gamma} u_{n-1}=0, \quad n \in \mathbb{N}_{0},
$$

a second-order difference equation with constant coefficients.

It is interesting that, by using the change

$$
u_{n}=\frac{v_{n}}{\gamma^{n}}
$$

(30) is transformed into the equation

$$
v_{n+1}-(\alpha+\delta) v_{n}+(\alpha \delta-\beta \gamma) v_{n-1}=0, \quad n \in \mathbb{N}_{0} .
$$

Let $\tilde{\lambda}_{1}$ and $\tilde{\lambda}_{2}$ be the characteristic roots of the characteristic polynomial:

$$
P(\lambda)=\lambda^{2}-(\alpha+\delta) \lambda+(\alpha \delta-\beta \gamma)
$$

associated with (32). Then by a well-known result on linear difference equations with constant coefficients and above transformations we get

$$
z_{n}=-\frac{\delta}{\gamma}+\frac{1}{\gamma} \cdot \frac{c_{1} \widetilde{\lambda}_{1}^{n+1}+c_{2} \widetilde{\lambda}_{2}^{n+1}}{c_{1} \widetilde{\lambda}_{1}^{n}+c_{2} \widetilde{\lambda}_{2}^{n}}
$$

if $(\alpha+\delta)^{2} \neq 4(\alpha \delta-\beta \gamma)$, and

$$
z_{n}=-\frac{\delta}{\gamma}+\frac{\widetilde{\lambda}_{1}}{\gamma} \cdot \frac{\widehat{c}_{1}+\widehat{c}_{2}(n+1)}{\widehat{c}_{1}+\widehat{c}_{2} n},
$$

if $(\alpha+\delta)^{2}=4(\alpha \delta-\beta \gamma)$.

In our case we have that $\alpha=0, \beta=1, \gamma=b$, and $\delta=a$, so that (30) becomes

$$
b u_{n+1}-a u_{n}-u_{n-1}=0, \quad n \in \mathbb{N}_{0},
$$

while the constant in (29) is equal to $-a / b$. The characteristic roots of the characteristic polynomial associated with (36) are

$$
\lambda_{1}=\frac{a+\sqrt{a^{2}+4 b}}{2 b}, \quad \lambda_{2}=\frac{a-\sqrt{a^{2}+4 b}}{2 b} .
$$

Hence, the general solution of (36) is

$$
u_{n}=c_{1}\left(\frac{a+\sqrt{a^{2}+4 b}}{2 b}\right)^{n}+c_{2}\left(\frac{a-\sqrt{a^{2}+4 b}}{2 b}\right)^{n},
$$

if $a^{2}+4 b \neq 0$, and

$$
u_{n}=\left(c_{1}+c_{2} n\right)\left(\frac{a}{2 b}\right)^{n}=\left(c_{1}+c_{2} n\right)\left(-\frac{2}{a}\right)^{n},
$$

if $a^{2}+4 b=0$.

If we choose initial values $u_{-1}$ and $u_{0}$ such that

$$
u_{-1}=1, \quad u_{0}=z_{-1}+\frac{a}{b},
$$

then from (40) and (38) we get

$$
u_{n}=\frac{\left(1+\lambda_{1}\left(b z_{-1}+a\right)\right) \lambda_{1}^{n}-\left(1+\lambda_{2}\left(b z_{-1}+a\right)\right) \lambda_{2}^{n}}{\sqrt{a^{2}+4 b}},
$$

$$
n \geq-1 \text {, }
$$

if $a^{2}+4 b \neq 0$, while from (40) and (39) we get

$$
u_{n}=\left(z_{-1}+\frac{a}{b}+n\left(z_{-1}+\frac{a}{b}+\frac{2}{a}\right)\right)\left(-\frac{2}{a}\right)^{n}, \quad n \geq-1 \text {, }
$$

if $a^{2}+4 b=0$ 
Using (41) and (42) in (27) we get

$$
z_{n}=-\frac{a}{b}+\frac{\left(1+\lambda_{1}\left(b z_{-1}+a\right)\right) \lambda_{1}^{n+1}-\left(1+\lambda_{2}\left(b z_{-1}+a\right)\right) \lambda_{2}^{n+1}}{\left(1+\lambda_{1}\left(b z_{-1}+a\right)\right) \lambda_{1}^{n}-\left(1+\lambda_{2}\left(b z_{-1}+a\right)\right) \lambda_{2}^{n}}
$$$$
n \geq-1
$$

if $a^{2}+4 b \neq 0$, and

$$
z_{n}=\frac{4}{a}-\frac{2}{a} \frac{\left(z_{-1}-4 / a+(n+1)\left(z_{-1}-2 / a\right)\right)}{\left(z_{-1}-4 / a+n\left(z_{-1}-2 / a\right)\right)},
$$

$$
n \geq-1
$$

if $a^{2}+4 b=0$.

Using the fact that the sequences $y_{n}^{(j)}=y_{n s+j}, n \geq-1$, $j=\overline{0, s-1}$, are solutions of (25), from formulas (43) and (44) it follows that

$$
y_{n}^{(j)}=-\frac{a}{b}+\frac{\left(1+\lambda_{1}\left(b y_{j-s}+a\right)\right) \lambda_{1}^{n+1}-\left(1+\lambda_{2}\left(b y_{j-s}+a\right)\right) \lambda_{2}^{n+1}}{\left(1+\lambda_{1}\left(b y_{j-s}+a\right)\right) \lambda_{1}^{n}-\left(1+\lambda_{2}\left(b y_{j-s}+a\right)\right) \lambda_{2}^{n}}, \quad n \geq-1,
$$

for $j=\overline{0, s-1}$, if $a^{2}+4 b \neq 0$, and

$$
\begin{array}{r}
y_{n}^{(j)}=\frac{4}{a}-\frac{2}{a} \frac{\left(y_{j-s}-4 / a+(n+1)\left(y_{j-s}-2 / a\right)\right)}{\left(y_{j-s}-4 / a+n\left(y_{j-s}-2 / a\right)\right)}, \\
n \geq-1,
\end{array}
$$

for $j=\overline{0, s-1}$, if $a^{2}+4 b=0$.

From (21) we have that

$$
x_{n}=y_{n} x_{n-k}, \quad n \geq-s,
$$

which implies that

$$
x_{k m_{1}+j_{1}}=y_{k m_{1}+j_{1}} x_{k\left(m_{1}-1\right)+j_{1}}=x_{j_{1}-k} \prod_{i=0}^{m_{1}} y_{k i+j_{1}},
$$

for $m_{1} \in \mathbb{N}_{0}$ and $j_{1}=-s,-s+1, \ldots,-s+k-1$.

Although formulas (45)-(48) give solutions to (15) they are not easily applicable due to the assumption $\operatorname{gcd}(k, s)=1$.
Namely, subsequences $\left(y_{n}^{(j)}\right), j=\overline{0, s-1}$, appearing in (45) and (46), and the subsequences $\left(y_{k m_{1}+j_{1}}\right), j_{1}=-s,-s+$ $1, \ldots,-s+k-1$, appearing in (48), are different decompositions of the sequence $\left(y_{n}\right)_{n \geq-s}$, so they are not easily connected to each other.

However, if $s=1$ in (15), then they give complete solution of the equation. Namely, in this case (45) and (46) become

$$
y_{n}=-\frac{a}{b}+\frac{\left(1+\lambda_{1}\left(b y_{-1}+a\right)\right) \lambda_{1}^{n+1}-\left(1+\lambda_{2}\left(b y_{-1}+a\right)\right) \lambda_{2}^{n+1}}{\left(1+\lambda_{1}\left(b y_{-1}+a\right)\right) \lambda_{1}^{n}-\left(1+\lambda_{2}\left(b y_{-1}+a\right)\right) \lambda_{2}^{n}},
$$

if $a^{2}+4 b \neq 0$, and

$$
y_{n}=\frac{4}{a}-\frac{2\left(y_{-1}-4 / a+(n+1)\left(y_{-1}-2 / a\right)\right)}{a\left(y_{-1}-4 / a+n\left(y_{-1}-2 / a\right)\right)}, \quad n \geq-1,
$$

if $a^{2}+4 b=0$.

Using (49) and (50) in (48), we get

$$
x_{k m_{1}+j_{1}}=x_{j_{1}-k} \prod_{i=0}^{m_{1}}\left(-\frac{a}{b}+\frac{\left(1+\lambda_{1}\left(b\left(x_{-1} / x_{-1-k}\right)+a\right)\right) \lambda_{1}^{k i+j_{1}+1}-\left(1+\lambda_{2}\left(b\left(x_{-1} / x_{-1-k}\right)+a\right)\right) \lambda_{2}^{k i+j_{1}+1}}{\left(1+\lambda_{1}\left(b\left(x_{-1} / x_{-1-k}\right)+a\right)\right) \lambda_{1}^{k i+j_{1}}-\left(1+\lambda_{2}\left(b\left(x_{-1} / x_{-1-k}\right)+a\right)\right) \lambda_{2}^{k i+j_{1}}}\right) \text {, }
$$

for each $j_{1} \in\{-1,0, \ldots, k-2\}$, and every $m_{1} \in \mathbb{N}_{0}$, if $a^{2}+$ $4 b \neq 0$, and

$$
x_{k m_{1}+j_{1}}=x_{j_{1}-k} \prod_{i=0}^{m_{1}}\left(\frac{4}{a}-\frac{2\left(\left(x_{-1} / x_{-1-k}\right)-4 / a+\left(k i+j_{1}+1\right)\left(\left(x_{-1} / x_{-1-k}\right)-2 / a\right)\right)}{a\left(\left(x_{-1} / x_{-1-k}\right)-4 / a+\left(k i+j_{1}\right)\left(\left(x_{-1} / x_{-1-k}\right)-2 / a\right)\right)}\right),
$$


for each $j_{1} \in\{-1,0, \ldots, k-2\}$, and every $m_{1} \in \mathbb{N}_{0}$, if $a^{2}+4 b=$ 0 .

\section{Asymptotic Behavior of Solutions of (15) When $s=1$}

In this section we will use formulas (51) and (52) in studying of the asymptotic behavior of solutions of (15) for the case when $s=1$.

The results are incorporated into two theorems. Before we formulate and prove the results we will present two auxiliary results.

Lemma 4. Assume that $s=1, a, b \in \mathbb{R} \backslash\{0\}$, and $a+b=1$. Then difference equation (15) has a (not necessarily prime) $k$ periodic solution.

Proof. Since $a+b=1$ it follows that $a^{2}+4 b \geq 0$ (this is equivalent to $\left.(1+b)^{2} \geq 0\right)$. Further, in this case (25) has one or two stationary solutions. Really, if $z_{n}=z, z \geq-1$, then it follows that $b z^{2}+a z-1=0$. That is, $b z^{2}+(1-b) z-1=$ $(b z+1)(z-1)=0$, from which it follows that

$$
z_{1}=1, \quad z_{2}=-\frac{1}{b}
$$

if $a^{2}+4 b>0$, that is, if $(1+b)^{2}>0$, and

$$
z_{1}=z_{2}=1
$$

if $a^{2}+4 b=0$, that is, if $b=-1$. Hence, in both cases

$$
z_{n}=1, \quad n \geq-1,
$$

is a stationary solution of (25).

By using (21), we see that, for the stationary solution of (25) given in (55), we have a solution of (15) such that

$$
x_{n}=x_{n-k}, \quad n \geq-1,
$$

from which the lemma follows.

Lemma 5. Assume that $s=1, a, b \in \mathbb{R} \backslash\{0\}$, and $b-a=1$. Then difference equation (15) has a (not necessarily prime) $2 k$ periodic solution.

Proof. First note that from the condition $b-a=1$ we have also that $a^{2}+4 b \geq 0$ (it is also equivalent to $(1+b)^{2} \geq 0$ ). On the other hand, in this case (25) also has one or two stationary solutions. Namely, if $z_{n}=z, z \geq-1$, then it follows that $b z^{2}+$ $a z-1=0$. That is, $b z^{2}+(b-1) z-1=(b z-1)(z+1)=0$, from which we obtain

$$
z_{1}=-1, \quad z_{2}=\frac{1}{b}
$$

if $a^{2}+4 b>0$, that is, if $(1+b)^{2}>0$, and

$$
z_{1}=z_{2}=-1
$$

if $a^{2}+4 b=0$, that is, if $b=-1$. Hence, in both cases

$$
z_{n}=-1, \quad n \geq-1
$$

is a stationary solution of (25).

By using (21), we see that the stationary solution of (25) given in (59) produces a solution of (15) such that $x_{n}=-x_{n-k}$, $n \geq-1$, which implies that

$$
x_{n}=x_{n-2 k}, \quad n \geq k-1,
$$

from which the lemma follows.

Remark 6. By some calculation it is easy to see that $-a / b+$ $\lambda_{1}=1$ if and only if $a+b=1$ and $b \geq-1$, while $-a / b+\lambda_{2}=1$ if and only if $a+b=1$ and $b \leq-1$.

Also, $-a / b+\lambda_{1}=-1$ if and only if $b-a=1$ and $b \leq-1$, while $-a / b+\lambda_{2}=-1$ if and only if $b-a=1$ and $b \geq-1$.

Theorem 7. Consider (15) with $s=1$. Assume that $a, b \in \mathbb{R} \backslash$ $\{0\}, a^{2}+4 b>0$, and $x_{-i} \neq 0, i=\overline{1, k+1}$. Let $\lambda:=\lambda_{i}$, where $\left|\lambda_{i}\right|=\max \left\{\left|\lambda_{1}\right|,\left|\lambda_{2}\right|\right\}, i \in\{1,2\}$. Then the following statements are true.

(a) If $|-a / b+\lambda|<1$ and $1+\lambda_{i}\left(b\left(x_{-1} / x_{-1-k}\right)+a\right) \neq 0$, for $i=1,2$, then $x_{n} \rightarrow 0$, as $n \rightarrow \infty$.

(b) If $\left|-a / b+\lambda_{1}\right|<1,1+\lambda_{1}\left(b\left(x_{-1} / x_{-1-k}\right)+a\right) \neq 0$, and $1+\lambda_{2}\left(b\left(x_{-1} / x_{-1-k}\right)+a\right)=0$, then $x_{n} \rightarrow 0$, as $n \rightarrow$ $\infty$.

(c) If $\left|-a / b+\lambda_{2}\right|<1,1+\lambda_{1}\left(b\left(x_{-1} / x_{-1-k}\right)+a\right)=0$, and $1+\lambda_{2}\left(b\left(x_{-1} / x_{-1-k}\right)+a\right) \neq 0$, then $x_{n} \rightarrow 0$, as $n \rightarrow \infty$.

(d) If $|-a / b+\lambda|>1$ and $1+\lambda_{i}\left(b\left(x_{-1} / x_{-1-k}\right)+a\right) \neq 0$, for $i=1,2$, then $\left|x_{n}\right| \rightarrow \infty$, as $n \rightarrow \infty$.

(e) If $\left|-a / b+\lambda_{1}\right|>1,1+\lambda_{1}\left(b\left(x_{-1} / x_{-1-k}\right)+a\right) \neq 0$, and $1+\lambda_{2}\left(b\left(x_{-1} / x_{-1-k}\right)+a\right)=0$, then $\left|x_{n}\right| \rightarrow \infty$, as $n \rightarrow \infty$.

(f) If $\left|-a / b+\lambda_{2}\right|>1,1+\lambda_{1}\left(b\left(x_{-1} / x_{-1-k}\right)+a\right)=0$, and $1+\lambda_{2}\left(b\left(x_{-1} / x_{-1-k}\right)+a\right) \neq 0,\left|x_{n}\right| \rightarrow \infty$, as $n \rightarrow \infty$.

(g) If $-a / b+\lambda=1$ and $1+\lambda_{i}\left(b\left(x_{-1} / x_{-1-k}\right)+a\right) \neq 0$, for $i=1,2$, then $x_{n}$ converges to a (not necessarily prime) $k$-periodic solution of (15), as $n \rightarrow \infty$.

(h) If $-a / b+\lambda_{1}=1,1+\lambda_{1}\left(b\left(x_{-1} / x_{-1-k}\right)+a\right) \neq 0$, and $1+$ $\lambda_{2}\left(b\left(x_{-1} / x_{-1-k}\right)+a\right)=0$, then $x_{n}$ is a (not necessarily prime) $k$-periodic solution of (15).

(i) If $-a / b+\lambda_{2}=1,1+\lambda_{1}\left(b\left(x_{-1} / x_{-1-k}\right)+a\right)=0$, and $1+$ $\lambda_{2}\left(b\left(x_{-1} / x_{-1-k}\right)+a\right) \neq 0$, then $x_{n}$ is a (not necessarily prime) $k$-periodic solution of (15).

(j) If $-a / b+\lambda=-1$ and $1+\lambda_{i}\left(b\left(x_{-1} / x_{-1-k}\right)+a\right) \neq 0$, for $i=1,2$, then $x_{n}$ converges to a (not necessarily prime) $2 k$-periodic solution of (15), as $n \rightarrow \infty$.

(k) If $-a / b+\lambda_{1}=-1,1+\lambda_{1}\left(b\left(x_{-1} / x_{-1-k}\right)+a\right) \neq 0$, and $1+\lambda_{2}\left(b\left(x_{-1} / x_{-1-k}\right)+a\right)=0$, then $x_{n}$ is a (not necessarily prime) $2 k$-periodic solution of (15). 
(1) If $-a / b+\lambda_{2}=-1,1+\lambda_{1}\left(b\left(x_{-1} / x_{-1-k}\right)+a\right)=0$, and $1+\lambda_{2}\left(b\left(x_{-1} / x_{-1-k}\right)+a\right) \neq 0$, then $x_{n}$ is a (not necessarily prime) $2 k$-periodic solution of (15).

Proof. First note that since $x_{-i} \neq 0, i=\overline{1, k+1}$, we have that $x_{n} \neq 0$ for every $n \geq-(k+1)$. Further note that the condition $a^{2}+4 b>0$ implies that $\lambda_{1}, \lambda_{2} \in \mathbb{R}, \lambda_{1} \neq \lambda_{2}$. If it were $\lambda_{1}=-\lambda_{2}$, then it would be $a=0$, the case which is excluded. Hence we have that $\left|\lambda_{1}\right| \neq\left|\lambda_{2}\right|$, from which it follows that

$$
\min \left\{\left|\lambda_{1}\right|,\left|\lambda_{2}\right|\right\}<\max \left\{\left|\lambda_{1}\right|,\left|\lambda_{2}\right|\right\}
$$

Let

$$
a_{m_{1}}^{\left(j_{1}\right)}:=-\frac{a}{b}+\frac{\left(1+\lambda_{1}\left(b\left(x_{-1} / x_{-1-k}\right)+a\right)\right) \lambda_{1}^{k m_{1}+j_{1}+1}-\left(1+\lambda_{2}\left(b\left(x_{-1} / x_{-1-k}\right)+a\right)\right) \lambda_{2}^{k m_{1}+j_{1}+1}}{\left(1+\lambda_{1}\left(b\left(x_{-1} / x_{-1-k}\right)+a\right)\right) \lambda_{1}^{k m_{1}+j_{1}}-\left(1+\lambda_{2}\left(b\left(x_{-1} / x_{-1-k}\right)+a\right)\right) \lambda_{2}^{k m_{1}+j_{1}}},
$$

for $m_{1} \in \mathbb{N}_{0}$ and $j_{1}=-1,0, \ldots, k-2$.

Then if $1+\lambda_{i}\left(b\left(x_{-1} / x_{-1-k}\right)+a\right) \neq 0$, for $i=1,2$, we have that for each $j_{1} \in\{-1,0, \ldots, k-2\}$

$$
\lim _{m_{1} \rightarrow \infty} a_{m_{1}}^{\left(j_{1}\right)}=-\frac{a}{b}+\lambda
$$

From (51) and (63) the statements in (a) and (d) easily follow.

If $1+\lambda_{1}\left(b\left(x_{-1} / x_{-1-k}\right)+a\right) \neq 0$ and $1+\lambda_{2}\left(b\left(x_{-1} / x_{-1-k}\right)+\right.$ $a)=0$, then we have that for each $j_{1} \in\{-1,0, \ldots, k-2\}$

$$
a_{m_{1}}^{\left(j_{1}\right)}=-\frac{a}{b}+\lambda_{1}
$$

from which along with (51) the statements in (b) and (e) easily follow.

If $1+\lambda_{1}\left(b\left(x_{-1} / x_{-1-k}\right)+a\right)=0$ and $1+\lambda_{2}\left(b\left(x_{-1} / x_{-1-k}\right)+\right.$ a) $\neq 0$, then we have that for each $j_{1} \in\{-1,0, \ldots, k-2\}$

$$
a_{m_{1}}^{\left(j_{1}\right)}=-\frac{a}{b}+\lambda_{2}
$$

from which along with (51) the statements in (c) and (f) follow. $(\mathrm{g}),(\mathrm{j})$ in this case we have that for sufficiently large $m_{1}$

$$
a_{m_{1}}^{\left(j_{1}\right)}=-\frac{a}{b}+\lambda+O\left(\left(\frac{\min \left\{\left|\lambda_{1}\right|,\left|\lambda_{2}\right|\right\}}{\max \left\{\left|\lambda_{1}\right|,\left|\lambda_{2}\right|\right\}}\right)^{k m_{1}}\right)
$$

for each $j_{1} \in\{-1,0, \ldots, k-2\}$, from which along with (51), Lemmas 4 and 5, and Remark 6 these two statements follow.

(h), (k) in this case we have that (64) holds, from which along with (51) these two statements follow.

(i), (l) in this case we have that (65) holds, from which along with (51) these two statements follow.

Theorem 8. Consider (15) with $s=1$. Assume that $a, b \in \mathbb{R} \backslash$ $\{0\}, a^{2}+4 b=0$, and $x_{-i} \neq 0, i=\overline{1, k+1}$. Then the following statements are true.
(a) If $|2 / a|<1$, then $x_{n} \rightarrow 0$, as $n \rightarrow \infty$.
(b) If $|2 / a|>1$, then $\left|x_{n}\right| \rightarrow \infty$, as $n \rightarrow \infty$.
(c) If $|a|=2$, then $x_{n} \rightarrow 0$, as $n \rightarrow \infty$.

Proof. In this case we have that $\lambda_{1}=\lambda_{2}=-2 / a$. Let

$$
b_{m_{1}}^{\left(j_{1}\right)}:=\frac{4}{a}-\frac{2\left(\left(x_{-1} / x_{-1-k}\right)-4 / a+\left(k m_{1}+j_{1}+1\right)\left(\left(x_{-1} / x_{-1-k}\right)-2 / a\right)\right)}{a\left(\left(x_{-1} / x_{-1-k}\right)-4 / a+\left(k m_{1}+j_{1}\right)\left(\left(x_{-1} / x_{-1-k}\right)-2 / a\right)\right)},
$$

for $m_{1} \in \mathbb{N}_{0}$ and $j_{1}=-1,0, \ldots, k-2$.

Then for each $j_{1} \in\{-1,0, \ldots, k-2\}$ we have that

$$
\lim _{m_{1} \rightarrow \infty} b_{m_{1}}^{\left(j_{1}\right)}=\frac{2}{a}
$$

if $x_{-1} / x_{-1-k} \neq 2 / a$, while

$$
b_{m_{1}}^{\left(j_{1}\right)}=\frac{2}{a}
$$

for $j_{1} \in\{-1,0, \ldots, k-2\}$ and $m_{1} \in \mathbb{N}_{0}$, if $x_{-1} / x_{-1-k}=2 / a$.

From (52), (68), and (69) the statements in (a) and (b) easily follow. (c) in this case we have that for sufficiently large $m_{1}$

$$
\begin{aligned}
a_{m_{1}}^{\left(j_{1}\right)} & =\frac{2}{a}-\frac{2}{a k m_{1}}+O\left(\frac{1}{m_{1}^{2}}\right) \\
& = \pm\left(1-\frac{1}{k m_{1}}+O\left(\frac{1}{m_{1}^{2}}\right)\right) \\
& = \pm e^{-\left(1 /\left(k m_{1}\right)\right)+O\left(1 / m_{1}^{2}\right)}
\end{aligned}
$$

for each $j_{1} \in\{-1,0, \ldots, k-2\}$, from which along with (52) and the fact that $\sum_{j=1}^{m_{1}}(1 / j) \rightarrow+\infty$ as $m_{1} \rightarrow \infty$, the statement easily follows. 


\section{Acknowledgments}

This work was funded by the Deanship of Scientific Research (DSR), King Abdulaziz University, under Grant no. (11130/1433 HiCi). The authors, therefore, acknowledge technical and financial support of KAU. ways. Since in the case $\gamma=0$ (26) is a linear first-order

\section{References}

[1] H. Levy and F. Lessman, Finite difference equations, Macmillan, New York, NY, USA, 1961.

[2] S. Stević, "More on a rational recurrence relation," Applied Mathematics E-Notes, vol. 4, pp. 80-85, 2004.

[3] C. Çinar, "On the positive solutions of the difference equation $x_{n+1}=\left(x_{n-1}\right) /\left(1+x_{n} x_{n-1}\right)$," Applied Mathematics and Computation, vol. 150, no. 1, pp. 21-24, 2004.

[4] M. Aloqeili, "Dynamics of a rational difference equation," Applied Mathematics and Computation, vol. 176, no. 2, pp. 768774, 2006.

[5] A. Andruch-Sobiło and M. Migda, "On the rational recursive sequence," Tatra Mountains Mathematical Publications, vol. 43, pp. 1-9, 2009.

[6] I. Bajo and E. Liz, "Global behaviour of a second-order nonlinear difference equation," Journal of Difference Equations and Applications, vol. 17, no. 10, pp. 1471-1486, 2011.

[7] G. Papaschinopoulos and G. Stefanidou, "Asymptotic behavior of the solutions of a class of rational difference equations," International Journal of Difference Equations, vol. 5, no. 2, pp. 233-249, 2010.

[8] S. Stević, "On the difference equation $x_{n}=x_{n-2} /\left(b_{n}+\right.$ $\left.c_{n} x_{n-1} x_{n-2}\right)$," Applied Mathematics and Computation, vol. 218, no. 8, pp. 4507-4513, 2011.

[9] S. Stević, "On a third-order system of difference equations," Applied Mathematics and Computation, vol. 218, no. 14, pp. 7649-7654, 2012.

[10] S. Stević, "A short proof of the Cushing-Henson conjecture," Discrete Dynamics in Nature and Society, vol. 2006, Article ID 37264, 5 pages, 2006.

[11] S. Stević, "On some solvable systems of difference equations," Applied Mathematics and Computation, vol. 218, no. 9, pp. 50105018, 2012.

[12] S. Stević, J. Diblík, B. Iričanin, and Z. Šmardad, "On a solvable system of rational difference equations," Journal of Difference Equations and Applications, 2013.

[13] S. Stević, "On the recursive sequence $x_{n+1}=\alpha_{n}+\left(x_{n-1} / x_{n}\right)$. II," Dynamics of Continuous, Discrete \& Impulsive Systems A, vol. 10, no. 6, pp. 911-916, 2003.

[14] S. Stević, "On the recursive sequence $x_{n+1}=\left(A / \prod_{i=0}^{k} x_{n-i}\right)+$ $\left(1 / \prod_{j=k+2}^{2(k+1)} x_{n-j}\right)$," Taiwanese Journal of Mathematics, vol. 7 , no. 2 , pp. 249-259, 2003.

[15] S. Stević, "Global stability of a difference equation with maximum," Applied Mathematics and Computation, vol. 210, no. 2, pp. 525-529, 2009. 


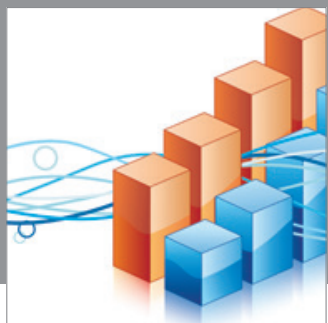

Advances in

Operations Research

mansans

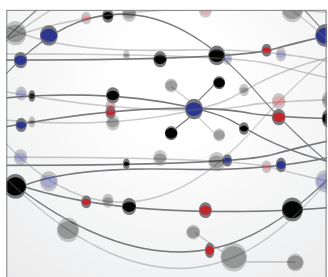

The Scientific World Journal
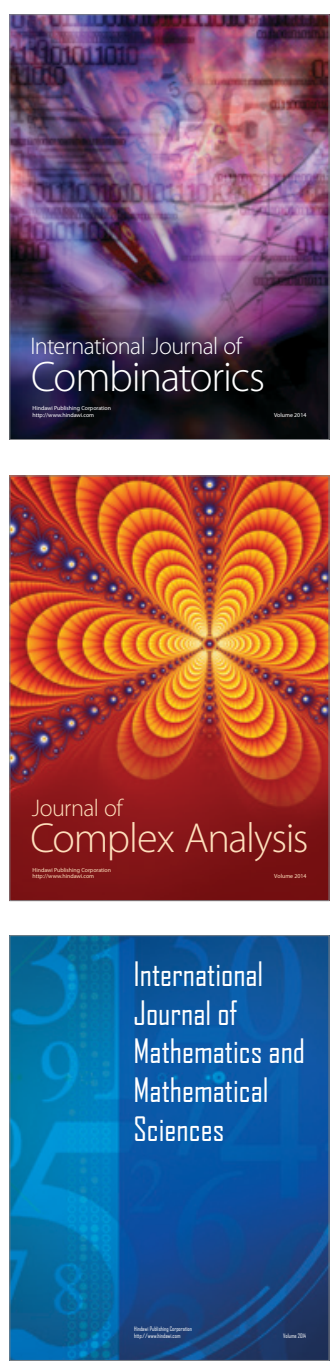
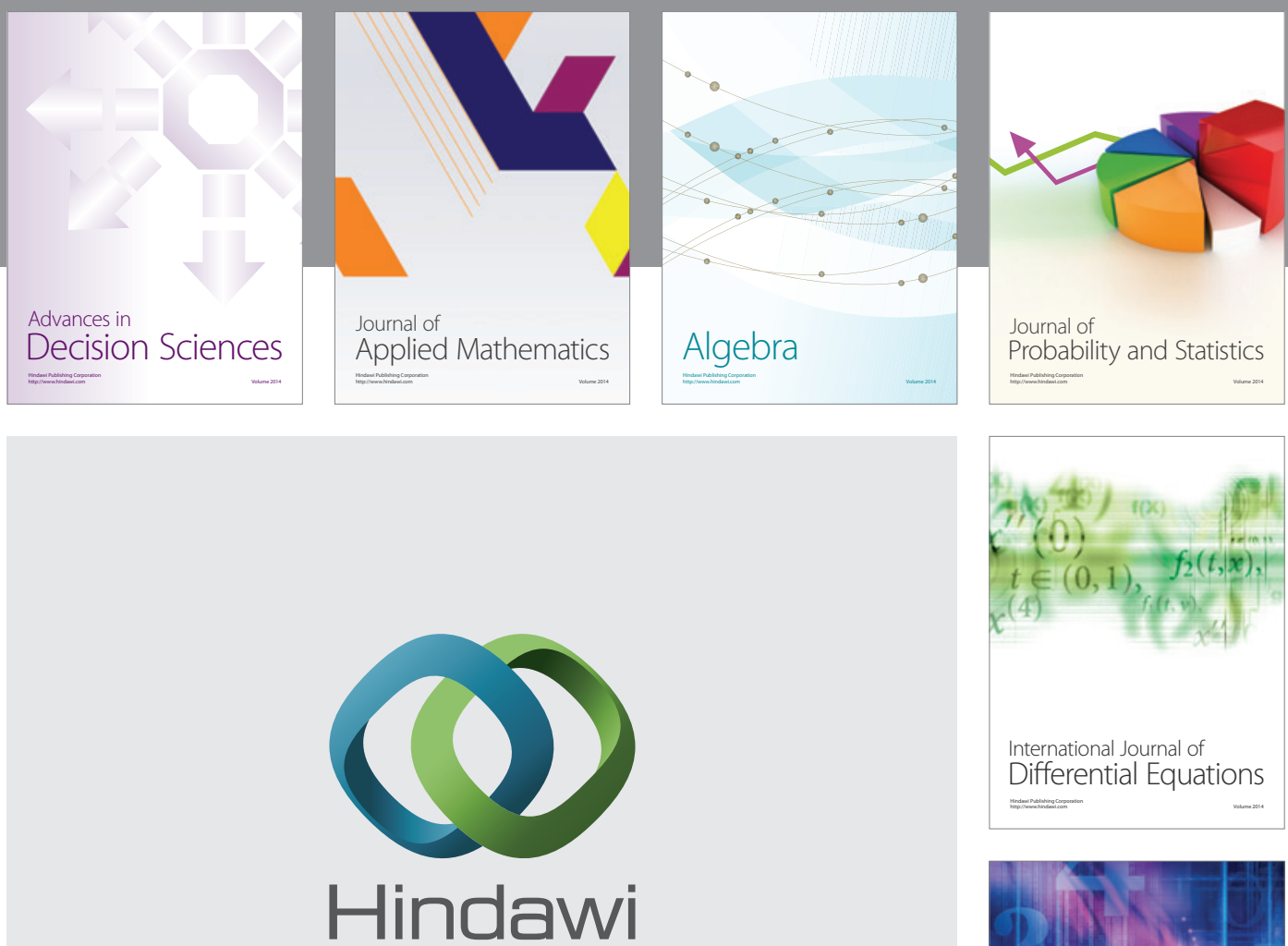

Submit your manuscripts at http://www.hindawi.com
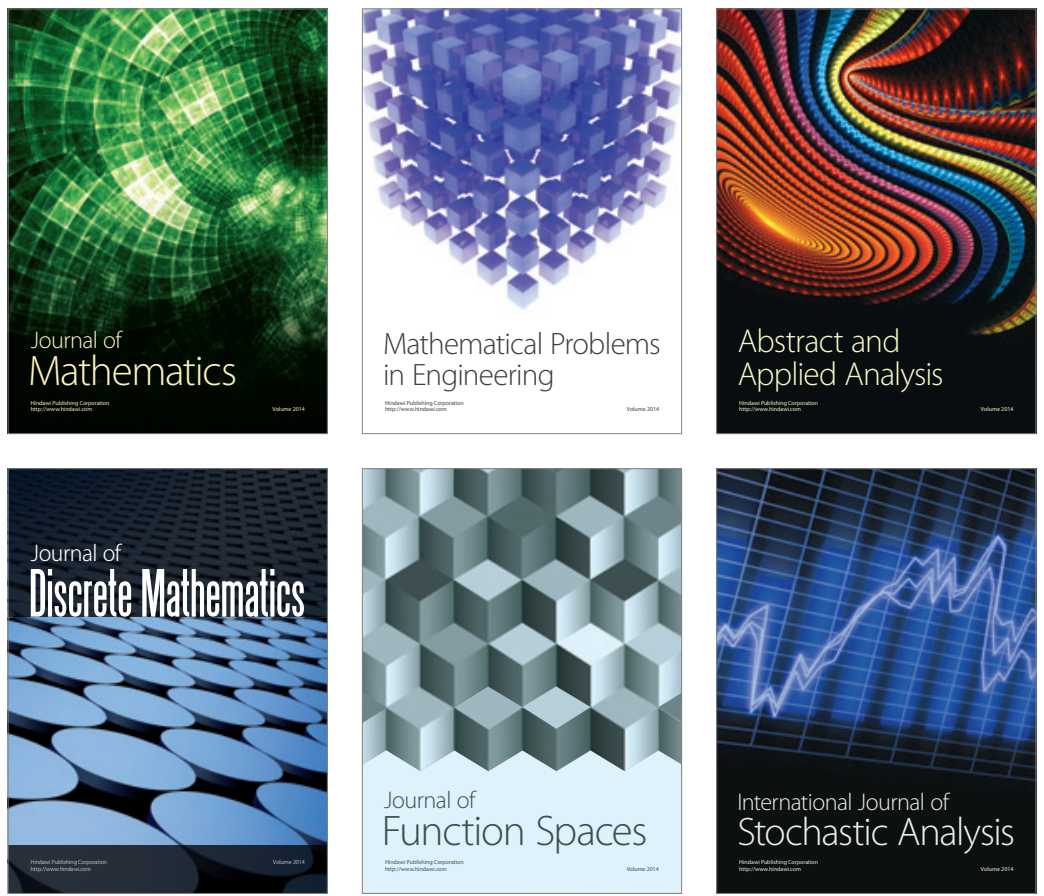

Journal of

Function Spaces

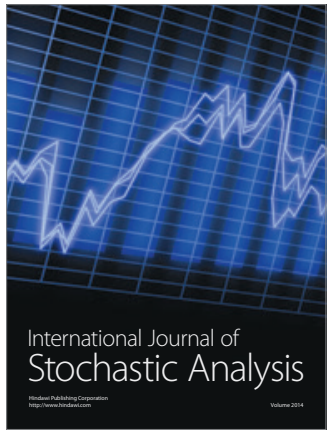

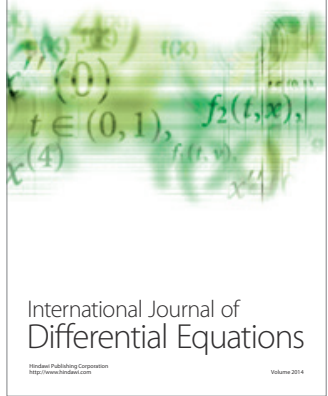
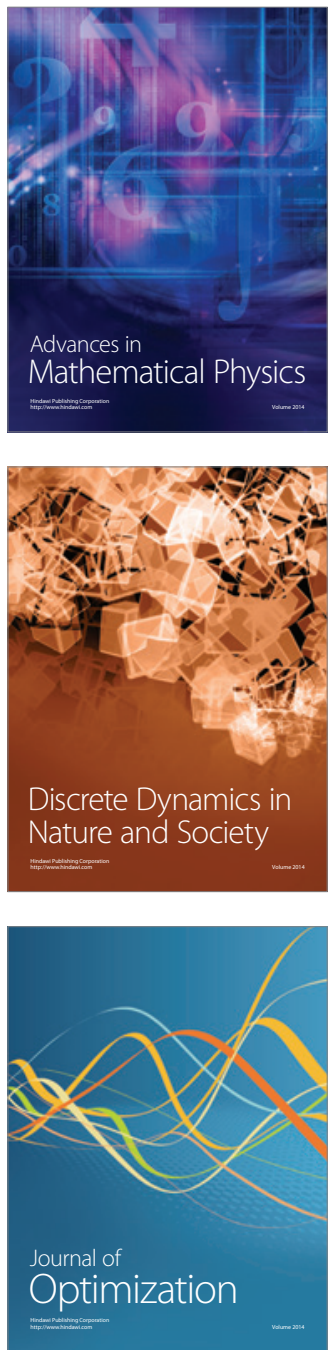